\title{
The problem of time in quantum mechanics
}

\author{
M. Bauer \\ Instituto de Física \\ Universidad Nacional Autónoma de México \\ bauer@fisica.unam.mx
}

August 16, 2016

\begin{abstract}
The problem of time in quantum mechanics concerns the fact that in the Schrödinger equation time is a parameter, not an operator. Pauli's objection to a time-energy uncertainty relation analogue to the positionmomentum one, conjectured by Heisenberg early on, seemed to exclude the existence of such an operator. However Dirac's formulation of electron's relativistic quantum mechanics (RQM) does allow the introduction of a dynamical time operator that is self-adjoint. Consequently, it can be considered as the generator of a unitary transformation of the system, as well as an additional system observable subject to uncertainty. In the present paper these aspects are examined within the standard framework of RQM.

Keywords: time operator; relativistic quantum mechanics; time-energy uncertainty relation

PACS: 03.65.-w ; 03.65.Ca ; 03.65.Pm
\end{abstract}

\section{Introduction}

In the time dependent Schrödinger equation (TDSE) of quantum mechanics (QM) time appears as a parameter, not as an operator 1, 2. Pauli's objection to the existence of a time operator, satisfying a commutation relation $[H, t]=-i \hbar$ as entertained by Heisenberg early on, is, to quote: "...from the C.R. written above it follows that $H$ possesses continously all eigenvalues from $-\infty$ to $+\infty$, whereas on the other hand, discrete eigenvalues of $H$ can be present. We, therefore, conclude that the introduction of an operator $t$ is basically forbidden and the time $t$ must necessarily be considered as on ordinary number ("c" number) in Quantum Mechanics".

Pauli's argument, sustained also by the fact that the system's stability requires the energy to have a finite minimum, has given rise to a variety of alternative proposals for a time-energy uncertainty relation and an extensive discussion 
of time in quantum mechanics throughout several decades [3, 4, 5, 6, 7, 8, , 9, 10, Within the time quantities considered one finds parametric (clock) time, tunneling times, decay times, dwell times, delay times, arrival times or jump times, i.e., both instantaneous values and intervals. To quote Ref.3: "In fact, the standard recipe to link the observables and the formalism does not seem to apply, at least in an obvious manner, to time observables". The extensive experimental confirmation of the Schrödinger equation asserts that this parameter corresponds to the time coordinate of the laboratory frame of reference, in both QM and RQM (Relativistic Quantum Mrchanics).

This is the problem of time in quantum mechanics. The questions to be answered are: 1) Can a time operator be found? 2) What is the status of a time-energy uncertainty relation? 3) How did the parameter $t$ enter into the Schrödinger equation?

Recently it has been shown that Dirac's formulation of relativistic quantum mechanics (RQM) does allow the introduction of a dynamical time operator that is self-adjoint 11. Consequently, it can be considered as the generator of a unitary transformation of the system, as well as an additional system observable subject to uncertainty. In the present paper these aspects are examined within the standard framework of RQM. The definition and main properties of the proposed time operator are recalled in Section 2. Section 3 analyses the effect of the corresponding unitary transformation. In Section 4 the ensuing timeenergy uncertaintinty relation is derived and shown how it circumvents Pauli's objection. It is also compared to the Mandelstam-Tamm formulation. Section 5 advances conclusions and possible developments.

Finally, to explain its presence in the TDSE has led to the consideration of time as an emergent property arising from the entanglement of a microscopic system with a classical environment in an overall closed time independent system, this property being apparent only to an internal observer [12, 13.

The proof that the commutation relation $[\hat{x}, \hat{p}]=i \hbar$ necessarily implies that the corresponding spectra of $\hat{x}$ and $\hat{p}$ go from $-\infty$ to $+\infty$ continously (as shown in Ref.14) is absent from most introductory textbooks on quantum mechanics. Also absent is the fact that the representations of the position and momentum operators in the configuration and momentum spaces follow from this commutation relation, as well as the fact that the representations of the state vector in configuration and momentum spaces are Fourier transform of each other. This is pedagogically unfortunate, as the student is induced to consider these as unrelated assumptions. In particular he will miss the connection of the infinite continuity of these espectra to unitary transformations and group properties, which is a cornerstone of the further development of quantum mechanics and of quantum field theory. Appendix A presents a unified description of the consequences of the commutation relation that could be incorporated in textbooks.. 


\section{The dynamical time operator in RQM[11]}

A dynamical self-adjoint "time operator"

$$
\hat{T}=\boldsymbol{\alpha} \cdot \hat{\mathbf{r}} / c+\beta \tau_{0}
$$

has been proposed in analogy to the Dirac free particle Hamiltonian $\hat{H}_{D}=$ $c \boldsymbol{\alpha} \cdot \hat{\mathbf{p}}+\beta m_{0} c^{2}$, where $\alpha_{i}(i=1,2,3)$ and $\beta$ are the $4 \times 4$ Dirac matrices, satisfying the anticonmutation relations:

$$
\alpha_{i} \alpha_{j}+\alpha_{j} \alpha_{i}=2 \delta_{i j} \quad \alpha_{i} \beta+\beta \alpha_{i}=0 \quad \beta^{2}=1
$$

The parameter $\tau_{0}$ represents in principle an internal property of the sysytem, to be determined. In the Heisenberg picture, the time evolution of the time operator is given by:

$$
\hat{T}(t)=\boldsymbol{\alpha}(t) \cdot \hat{\mathbf{r}}(t) / c+\beta(t) \tau_{0}=\hat{T}(0)+\left(c \hat{\mathbf{p}} / \hat{H}_{D}\right)^{2} t+\text { oscillating terms }
$$

where use has been made of the following relations [14, 15, 16]:

$$
\begin{gathered}
\boldsymbol{\alpha}(t)=\boldsymbol{\alpha}(0)+\left\{\boldsymbol{\alpha}(0)-c \hat{\mathbf{p}} / \hat{H}_{D}\right\}\left\{\exp \left(-2 i \hat{H}_{D} t / \hbar\right)-1\right\} \\
\beta(t)=\beta(0)+\left\{\beta(0)-m_{0} c^{2} / \hat{H}_{D}\right\}\left\{\exp \left(-2 i \hat{H}_{D} t / \hbar\right)-1\right\} \\
\hat{\mathbf{r}}(t)=\hat{\mathbf{r}}(0)+\left(c^{2} \hat{\mathbf{p}} / \hat{H}_{D}\right) t+i\left(c \hbar / 2 \hat{H}_{D}\right)\left\{\exp \left(-2 i \hat{H}_{D} t / \hbar\right)-1\right\} \\
c \boldsymbol{\alpha}(0) .\left(c \hat{\mathbf{p}} / \hat{H}_{D}\right)=\left[\frac{d \hat{\mathbf{r}}}{d t}\right]_{t=0} .\left(c \hat{\mathbf{p}} / \hat{H}_{D}\right)=\left(c \hat{\mathbf{p}} / \hat{H}_{D}\right)^{2}+\text { oscillating terms }
\end{gathered}
$$

Thus $\hat{T}(t)$ exhibits a linear dependence on $t$ with a superimposed oscillation (Zitterbewegung), as occurs with the time development of the postion operator $\hat{\mathbf{r}}(t)$.

Following step by step the algebra developed for the Dirac Hamiltonian[14, 15, 16, the eigenvalue equation

$$
\hat{T}|\tau>=\tau| \tau>
$$

yields:

$$
\tau= \pm \tau_{r}= \pm\left[(r / c)^{2}+\tau_{0}^{2}\right]^{1 / 2}
$$

as $\hat{T}^{2}=(\hat{\mathbf{r}} / c)^{2}+\tau_{0}^{2}$. The eigenvalue spectrum has two continous branches, a positive and a negative one separated by a $2 \tau_{0}$ gap. As $r$ goes from $-\infty$ to $+\infty$ the positive branch drops from $+\infty$ to $\tau_{0}$ when $r=0$, and then rises to $+\infty$ again. The negative branch follows the opposite behaviour.

Each of these eigenvalues is doubly degenerate with respect to the component $\boldsymbol{\sigma} \cdot \hat{\boldsymbol{r}} / 2 r$ of the spin along the $\boldsymbol{r}$ direction, which commutes with $\hat{T}$. Thus one 
can find simultaneous eigenfunctions of $\boldsymbol{\sigma} \cdot \hat{\boldsymbol{r}} / 2 r$ and $\hat{T}$, giving rise to altogether four eigenvalue pairs $|\tau, \sigma\rangle$. The "time eigenvectors" are:

$$
\left| \pm \tau_{r}, \pm 1 / 2>=u_{r}^{i}\right| \boldsymbol{r}>\quad i=1,2,3,4
$$

where $\mid \boldsymbol{r}>$ is the eigenvector of the position operator $\hat{\boldsymbol{r}}$ and $u_{r}^{i}$ is a four component spinor independent of the linear momentum $\boldsymbol{p}$. The four orthonormal spinors $u_{r}^{i}$ are listed in Ref.11.

In this formulation, $\tau_{0}$ plays the role of an invariant quantity in the $(\boldsymbol{r}, \tau)$ space, i.e., $\tau_{0}^{2}=\tau^{2}-(\boldsymbol{r} / c)^{2}$, as $m_{0} c^{2}$ plays in the $(\boldsymbol{p}, E)$ space, namely $\left(m_{0} c^{2}\right)^{2}=$ $E^{2}-(c \boldsymbol{p})^{2}$. To mantain the fundamental indeterminacy modulo $n 2 \pi$ ( $n$ an integer) in the phase of the complex eigenfunctions one has to set, for $n=1$ :

$$
\tau_{0}=2 \pi \hbar /<\beta>\varepsilon=h / m_{0} c^{2}
$$

This is the de Broglie period[18, 19]. Together with the Compton wave length, it sets a unified spacetime Compton scale that limits the wave packets width in space and time before negative energy and negative time components (particle

and antiparticle) occur significantly. Moreover, it supports the existence of an internal property, the "de Broglie clock" with a period $\tau_{0}=h / m_{0} c^{2}[20,21,22$.

\section{The time operator as generator of a unitary transformation}

The proposed operator is clearly self-adjoint and therefor can be the generator of a unitary transformation (Stone's theorem[17]):

$$
\hat{U}_{T}(\varepsilon)=e^{-i \varepsilon \hat{T} / \hbar}=e^{-i \varepsilon\left\{\boldsymbol{\alpha} \cdot \hat{\mathbf{r}} / c+\beta \tau_{0}\right\} / \hbar}
$$

where $\varepsilon$ is real and has the dimensions of energy.

For infinitesimal transformations $(\delta \varepsilon<<1)$, one can write:

$$
\hat{U}_{T}(\varepsilon) \simeq e^{-i(\delta \varepsilon)\{\boldsymbol{\alpha} \cdot \hat{\mathbf{r}} / c\} / \hbar} e^{-i(\delta \varepsilon) \beta \tau_{0} / \hbar}=e^{-i(\delta \varepsilon) \beta \tau_{0} / \hbar} e^{-i(\delta \varepsilon)\{\boldsymbol{\alpha} \cdot \hat{\mathbf{r}} / c\} / \hbar}
$$

as $\left[i(\delta \varepsilon)(\boldsymbol{\alpha} . \hat{\mathbf{r}} / c \hbar), i(\delta \varepsilon) \beta \tau_{0} / \hbar\right] \propto(\delta \varepsilon)^{2} \approx 0$ (Glauber proof[16, p.442]). Then the transformed Hamiltonian can be approximated as:

$$
\tilde{H}_{D}=\hat{U} \hat{H}_{D} \hat{U}^{\dagger} \simeq e^{i(\delta \varepsilon) \beta m_{0} c^{2} / \hbar} e^{i(\delta \varepsilon) \boldsymbol{\alpha} \cdot \hat{\mathbf{r}} / c \hbar} \hat{H}_{D} e^{-i(\delta \varepsilon) \boldsymbol{\alpha} \cdot \hat{\mathbf{r}} / c \hbar} e^{-i(\delta \varepsilon) \beta m_{0} c^{2} / \hbar}
$$

Consider first:

$$
\begin{aligned}
\tilde{h}_{D} & \doteq e^{i(\delta \varepsilon) \boldsymbol{\alpha} \cdot \hat{\mathbf{r}} / c \hbar} \hat{H}_{D} e^{-i(\delta \varepsilon) \boldsymbol{\alpha} \cdot \hat{\mathbf{r}} / c \hbar} \simeq \\
& \simeq\{I+i(\delta \varepsilon) \boldsymbol{\alpha} \cdot \hat{\mathbf{r}} / c \hbar+. .\} \hat{H}_{D}\{I-i(\delta \varepsilon) \boldsymbol{\alpha} \cdot \hat{\mathbf{r}} / c \hbar+. .\} \\
& \simeq \hat{H}_{D}+i\{(\delta \varepsilon) / c \hbar\}\left[\boldsymbol{\alpha} \cdot \hat{\mathbf{r}}, \hat{H}_{D}\right]+\ldots .
\end{aligned}
$$


Then using[14]:

$$
\left[\boldsymbol{\alpha} . \hat{\mathbf{r}}, \hat{H}_{D}\right]=3 i c \hbar I+2 \hat{H}_{D}\left\{\boldsymbol{\alpha}-c \hat{\mathbf{p}} / \hat{H}_{D}\right\} . \hat{\mathbf{r}}
$$

and substituting $3 I=\boldsymbol{\alpha} . \boldsymbol{\alpha}$, one obtains:

$$
\begin{aligned}
& \tilde{h}_{D} \simeq \hat{H}_{D}[\hat{\mathbf{p}}]+(\delta \varepsilon) \boldsymbol{\alpha} \cdot \boldsymbol{\alpha}+i\{(\delta \varepsilon) / c \hbar\} 2\left\{\hat{H}_{D}[\hat{\mathbf{p}}] \boldsymbol{\alpha} \cdot \hat{\mathbf{r}}-c \hat{\mathbf{p}} \cdot \hat{\mathbf{r}}\right\}= \\
= & c \boldsymbol{\alpha} \cdot\{\hat{\mathbf{p}}+(\delta \varepsilon) \boldsymbol{\alpha} / c\}+\beta m_{0} c^{2}+i 2\{(\delta \varepsilon) / c \hbar\}\left\{\hat{H}_{D}[\hat{\mathbf{p}}] \boldsymbol{\alpha} \cdot \hat{\mathbf{r}}-c \hat{\mathbf{p}} \cdot \hat{\mathbf{r}}\right\}= \\
= & \hat{H}_{D}[\hat{\mathbf{p}}+(\delta \varepsilon) \boldsymbol{\alpha} / c]+i 2\{(\delta \varepsilon) / c \hbar\}\left\{\hat{H}_{D}[\hat{\mathbf{p}}] \boldsymbol{\alpha} \cdot \hat{\mathbf{r}}-c \hat{\mathbf{p}} \cdot \hat{\mathbf{r}}\right\}
\end{aligned}
$$

Thus, the unitary transformation induces a shift in momentum by the amount:

$$
\delta \hat{\mathbf{p}}=\{(\delta \varepsilon) / c\} \boldsymbol{\alpha}=\left\{(\delta \varepsilon) / c^{2}\right\} c \boldsymbol{\alpha}
$$

as well as a Zitterbewegung behavior in the corresponding propagator $U(t)=$ $e^{-i \tilde{H}_{D} t / \hbar}$.

For repeated infinitessimal applications one obtains a momentum displacement $\Delta \hat{\mathbf{p}}$ whose expectation value is

$$
<\Delta \hat{\mathbf{p}}>=\left(\varepsilon / c^{2}\right) \mathbf{v}_{g p}=\gamma m_{0} \mathbf{v}_{g p}
$$

where $\gamma=\left\{1-\left(v_{g p} / c\right)^{2}\right\}^{-1 / 2}$ is the Lorentz factor and $\mathbf{v}_{g p}$ the group velocity.

It also induces a phase shift. Indeed:

$$
\left\langle\Psi\left|\tilde{H}_{D}[\mathbf{p}]\right| \Psi\right\rangle=\left\langle\Psi\left|e^{i(\delta \varepsilon) \beta \tau_{0} / \hbar} \tilde{h}_{D} e^{-i(\delta \varepsilon) \beta \tau_{0} / \hbar}\right| \Psi\right\rangle=\left\langle\Phi\left|\hat{H}_{D}[\mathbf{p}+\boldsymbol{\alpha} \delta \varepsilon / c]\right| \Phi\right\rangle+\ldots
$$

where

$$
|\Phi\rangle=e^{-i(\delta \varepsilon) \beta \tau_{0} / \hbar}|\Psi\rangle
$$

The phase shift is $\delta \varphi=(\delta \varepsilon) \beta \tau_{0} / \hbar$. For a finite transformation, its expectation value is

$$
\left.\langle\Delta \varphi\rangle=\left\{(\Delta \varepsilon) \tau_{0} / \hbar\right\}\langle\beta\rangle= \pm(1 / \gamma) m_{0} c^{2} \tau_{0} / \hbar\right)
$$

as $\left.\langle\beta\rangle=m_{0} c^{2} /<H_{D}\right\rangle= \pm m_{0} c^{2} / \varepsilon= \pm 1 / \gamma$, for a positive (negative) energy wave packet that contains both positive and negative energy free particle solutions [14. Thus the sign of $\langle\beta\rangle$ distinguishes the positive or negative energy branch where the momentum displacement takes place. To mantain the fundamental indeterminacy modulo $n 2 \pi$ ( $n$ an integer) in the phase of the complex eigenfunctions one has to set, for $n=1$ :

$$
\tau_{0}=2 \pi \hbar /<\beta>\varepsilon=h / m_{0} c^{2}
$$

This is the de Broglie period. One has then:

$$
h / p=h / \gamma m_{0} v_{g p}=h c^{2} / \gamma m_{0} c^{2} v_{g p}=(h / \varepsilon)\left(c^{2} / v_{g p}\right)=(1 / \nu) v_{p h}
$$

which is precisely the de Broglie wave length, that is, the product of the phase velocity by the period derived from the Planck relation $\varepsilon=h \nu$, as originally assumed by de Broglie 18 .

In conclusion, the dynamical time operator $T=\boldsymbol{\alpha} . \hat{\mathbf{r}} / c+\beta\left(h / m_{0} c^{2}\right.$ ) (where the parameter $\tau_{0}$ is equated to de Broglie period $\left.h / m_{0} c^{2}\right)$, generates the Lorentz 
boost that gives rise to the de Broglie wave. Indeed the fact that a rest frame oscillation gives rise to a travelling wave has been shown to be a simple consequence of special relativity applied to the complex-phase oscillation of stationnary states through the Lorentz transformation of the time dependence 18, 19, 20. To quote Baylis: "What in the rest frame is a synchronized oscillation in time is seen in the laboratory to be a wave in space".

What about Pauli's objection? The continous displacement in momentum implies also a continous displacement in energy only within the positive and the negative branches. No crossing of the energy gap is involved. Thus Pauli's objection is circumvented.

Finally, it is also interesting to note the following. In the same way as above, in the case of an infitesimal time lapse $(\delta t<<1)$ the unitary operator $U_{H_{D}}(\delta t)=e^{i(\delta t)\left\{c \boldsymbol{\alpha} \cdot \hat{\mathbf{p}}+\beta m_{0} c^{2}\right\} / \hbar}$ can be approximated as:

$$
U(\delta t) \simeq e^{i(\delta t)\{c \boldsymbol{\alpha} \cdot \hat{\mathbf{p}} / \hbar\}} e^{i(\delta t)\left\{\beta m_{0} c^{2} / \hbar\right\}}
$$

In configuration space this yields a displacement $\delta \mathbf{r}=\langle\hat{\mathbf{r}}+c \boldsymbol{\alpha}(\delta t)\rangle=\langle\hat{\mathbf{r}}\rangle+$ $\mathbf{v}_{g p .}(\delta t)$ and a phase shift $\delta \varphi=(\delta t)<\beta>m_{0} c^{2} / \hbar$. For repeated infinitessimal time displacements to reach $\Delta t=\gamma \tau_{0}=\gamma h / m_{0} c^{2}$ (the boosted de Broglie period), the phase shift is

$$
\Delta \varphi=\left(\gamma h / m_{0} c^{2}\right)\left(m_{0} c^{2} /<H>\right) m_{0} c^{2} / \hbar=h / \hbar=2 \pi
$$

These results are in agreement with the fact that the Hamiltonian is actually the generator of the time development of a system described by a wave packet. The approximate treatment provides only the displacement, neglecting the dispersion of the wave.

\section{The time-energy uncertainty relation}

The time operator and the Dirac Hamiltonian satisfy the commutaion relation:

$$
\left[\hat{T}, \hat{H}_{D}\right]=i \hbar\{I+2 \beta K\}+2 \beta\left\{\tau_{0} \hat{H}_{D}-m_{0} c^{2} \hat{T}\right\}
$$

where $K=\beta\left(2 \mathbf{s} . \mathbf{l} / \hbar^{2}+1\right)$ is a constant of motion 14. In the usual manner an uncertainty relation follows, namely:

$$
(\Delta T)\left(\Delta H_{D}\right) \geq(\hbar / 2)|\{1+2<\beta K>\}|=(\hbar / 2) \mid\left\{3+4\left\langle\text { s.l } / \hbar^{2}\right\rangle\right\} \mid
$$

To be noted now is that, with the present definition of the time operator, its uncertainty is related to the uncertainty in position $\Delta \hat{\mathbf{r}}$, in the same way as the energy uncertainty is related to the momentum uncertainty $\Delta \hat{\mathbf{p}}$. Indeed:

$$
\begin{aligned}
(\Delta T)^{2} & =\left\langle\hat{T}^{2}\right\rangle-\langle\hat{T}\rangle^{2}=\left\langle\hat{\mathbf{r}}^{2} / c^{2}+\tau_{0}^{2}\right\rangle-\langle\hat{T}\rangle^{2}= \\
& =\left\{(\Delta \mathbf{r})^{2}+\langle\hat{\mathbf{r}}\rangle^{2}\right\} / c^{2}+\tau_{0}^{2}-\langle\hat{T}\rangle^{2} \\
& =\left\{(\Delta \mathbf{r})^{2}+\langle\hat{\mathbf{r}}\rangle^{2}\right\} / c^{2}+\tau_{0}^{2}-\left\langle\boldsymbol{\alpha} \cdot \hat{\mathbf{r}} / c+\beta \tau_{0}\right\rangle^{2} \geq(\Delta \mathbf{r})^{2} / c^{2}
\end{aligned}
$$


and

$$
\begin{aligned}
\left(\Delta H_{D}\right)^{2} & =\left\langle\hat{H}_{D}^{2}\right\rangle-\left\langle\hat{H}_{D}\right\rangle^{2}= \\
& =c^{2}\left\{(\Delta \mathbf{p})^{2}+\langle\hat{\mathbf{p}}\rangle^{2}\right\}+\left(m_{0} c^{2}\right)^{2}-\left\langle c \boldsymbol{\alpha} \cdot \hat{\mathbf{p}}+\beta m_{0} c^{2}\right\rangle^{2} \geq c^{2}(\Delta \mathbf{p})^{2}
\end{aligned}
$$

Then

$$
(\Delta \hat{T})(\Delta \hat{H}) \gtrsim(\Delta \mathbf{r})(\Delta \mathbf{p}) \geq(3 \hbar / 2)
$$

This corresponds to Bohr's interpretation: the width of a wave packet, complementary to its momentum dispersion, measures the uncertainty in the time of passage at a certain point, and is thereby complementary to its energy dispersion.

\subsubsection{The Mandelstam-Tamm uncertainty relation[16, p.319]}

As an observable, the time operator can be subject to the Mandelstam-Tamm (MT) formulation of a time-energy uncertainty relation within standard QM, to wit: any observable $\hat{O}$ represented by a self-adjoint operator $\hat{O}$ not explicitly dependent on time, satisfies the dynamical equation:

$$
(i \hbar) \frac{d}{d t}<\hat{O}>=<[\hat{O}, \hat{H}]>
$$

From the commutator $[\hat{O}, \hat{H}]$ it follows that the uncertainties defined $\Delta \hat{O}$ and $\Delta \hat{H}$ satisfy the relation:

$$
(\Delta \hat{O})(\Delta \hat{H}) \geq(1 / 2)|<[\hat{O}, \hat{H}]>|=(1 / 2)\left|\frac{d}{d t}<\hat{O}>\right|
$$

Then, associated to any system observable $\hat{O}$, a related time uncertainty is defined as:

$$
\Delta \hat{T}_{\hat{O}}^{M T}=\frac{\Delta \hat{O}}{\left|\frac{d}{d t}<\hat{O}>\right|}
$$

From Eqs.29 and 30, it then follows that:

$$
\left(\Delta \hat{T}_{\hat{O}}^{M T}\right)(\Delta \hat{H}) \geq(\hbar / 2)
$$

This is the Mandelstam-Tamm time-energy uncertainty relation. $\Delta \hat{T}_{\hat{O}}^{M T}$ can be interpreted as "the time required for the center $\langle\hat{O}\rangle$ of this distribution to be displaced by an amount equal to its width $\Delta \hat{O}$ "[16].

Now let $\hat{O}$ be the dynamical time operator in RQM $\hat{T}=(\boldsymbol{\alpha} . \mathbf{r}) / c+\beta \tau_{0}$. Then, from Eq. 32:

$$
\Delta T_{\hat{T}}^{M T} \approx \frac{\Delta \hat{T}}{|\langle I+2 \beta K\rangle|}
$$


It follows that:

$$
\frac{\Delta \hat{T}}{\langle I+2 \beta K\rangle}\left(\Delta \hat{H}_{D}\right) \geq(\hbar / 2)
$$

or

$$
(\Delta \hat{T})\left(\Delta \hat{H}_{D}\right) \geq(\hbar / 2)|\langle I+2 \beta K\rangle|
$$

In the non relativistic limit $\left\langle\hat{H}_{D}\right\rangle \simeq m_{0} c^{2}$, neglecting the oscillating terms,:

$$
\hat{T}(t) \simeq \tau_{0}+\left(c p / m_{0} c^{2}\right)^{2} t+\ldots
$$

Thus:

$$
\frac{d<\hat{T}>}{d t}=\left\langle\left(c p / m_{0} c^{2}\right)^{2}\right\rangle=\left(v_{g p} / c\right)^{2}
$$

and

$$
\Delta T_{\hat{T}}^{M T} \simeq \frac{\Delta \hat{T}}{\left(v_{g p} / c\right)^{2}} \gg \Delta \hat{T}
$$

as $v_{g p}<<c$. The uncertainty of the Mandelstam-Tamm time operator associated with the observable $\hat{T}$ overestimates largely the usual uncertainty.

In the ultra relativistic limit $\left\langle\hat{H}_{D}\right\rangle \simeq c p$ :

$$
\hat{T}(t) \simeq t+\left(m_{0} c^{2} / c p\right)^{2} \tau_{0}+\ldots \simeq t
$$

and

$$
\Delta T_{\hat{T}}^{M T} \simeq \Delta \hat{T}
$$

\section{Conclusion}

A self-adjoint internal "time operator" can be defined within Dirac's formulation of relativistic quantum mechanics (RQM). As with all observables, it is accorded in the Heisenberg picture a dependence on the external laboratory time parameter $t$ in the TDSE, which is atributed to the entanglement of the microscopic system with a classical environment. Also as an observable, it is in general subject to an uncertainty shown to be proportional to the position uncertainty; and consequently to a time-energy uncertainty relation where the energy uncertainty is related to the momentum one, supporting Bohr's original interpretation as the uncertainty in the instant of passage at a point of the trajectory. It nevertheless circumvents Pauli's objection, due to the fact that, as generator of a unitary transformation, it actually produces displacements in the continous momentum spectrum, and thus only indirectly in energy. This resolves the problem of time in quantum mechanics.

Based on the position observable, the time operator is expected to exhibit a Zitterbewegung behaviour about its linear dependence on $t$. As occurs with the position Zitterbewegung, its observation is be beyond current 
technical possibilities. However it may be observable in systems that simulate Dirac's Hamiltonian, where position Zitterbewegung has allready been exhibited experimentally [23, 24, 25]. A corresponding time operator can be constructed in each case and its properties examined.

Finally, general relativity accords a dynamical behaviour to space-time, firmly confirmed recently by the detection of gravitational waves. As a dynamical time is definitively incompatible with a time parameter, this becomes from the start a fundamental "problem of time" in quantum gravity [26, 27, 28. Whether the time operator here introduced has a relevance in this subject, is a venue to be considered[29].

\section{Appendix A. The lore of $[\hat{x}, \hat{p}]=i \hbar$}

For pedagogical purposes this Appendix collects all the properties derivable from the commutation relation, that are usually dispersed in quantum mechanical books.

To represent observables the operators . $\hat{x}$.and. $\hat{p}$.are self-adjoint $\left(\hat{x}=\hat{x}^{\dagger}\right.$, $\hat{p}=\hat{p}^{\dagger}$ ), which insures real eigenvalues. Then:

1) Spectrum

Consider the eigenvalue equation:

$$
\hat{x}|x\rangle=x|x\rangle
$$

By Stone-von Newmann's theorem 17, 16 the operator $U(\alpha)=\exp (-i \alpha \hat{p} / \hbar)$ with $a$ real is unitary. Then:

$$
\begin{aligned}
& \hat{x}\{U(\alpha)|x\rangle\} \\
= & \hat{x}\left\{1+(1 / 1 !)(-i \alpha \hat{p} / \hbar)+(1 / 2 !)(-i \alpha \hat{p} / \hbar)^{2}+(1 / 3 !)(-i \alpha \hat{p} / \hbar)^{3}+\ldots\right\}|x\rangle \\
= & \left(\hat{x}+(-i \alpha / \hbar) \hat{x} \hat{p}+(1 / 2)(-i \alpha / \hbar)^{2} \hat{x} \hat{p}^{2}+\ldots\right)|x\rangle \\
= & \left(\hat{x}+(-i \alpha / \hbar)\{\hat{p} \hat{x}+[\hat{x}, \hat{p}]\}+(1 / 2)(-i \alpha / \hbar)^{2}\left\{\hat{p}^{2} \hat{x}+\left[\hat{x}, \hat{p}^{2}\right]\right\}+\ldots\right)|x\rangle \\
= & \left(\hat{x}+(-i \alpha / \hbar)\{\hat{p} \hat{x}+i \hbar\}+(1 / 2)(-i \alpha / \hbar)^{2}\left\{\hat{p}^{2} \hat{x}+2 i \hbar \hat{p}\right\}+\ldots\right)|x\rangle \\
= & \left\{1+(-i \alpha \hat{p} / \hbar)+(1 / 2)(-i \alpha \hat{p} / \hbar)^{2}+\ldots\right\}(\hat{x}+\alpha)|x\rangle \\
= & (x+\alpha)\left\{1+(-i \alpha \hat{p} / \hbar)+(1 / 2)(-i \alpha \hat{p} / \hbar)^{2}+\ldots\right\}|x\rangle=(x+\alpha)\{U(\alpha)|x\rangle\}
\end{aligned}
$$

One concludes that:

$$
\{U(\alpha)|x\rangle\}=|x+\alpha\rangle
$$

As $\alpha$ is arbitrary, it follows that the eigenvalues of $\hat{x}$ are continous from $-\infty$ a $+\infty$, and that the eigenvectors satisfy:

$$
\left\langle x^{\prime} \mid x\right\rangle=\delta\left(x^{\prime}-x\right) \quad \quad \quad \int d x|x\rangle\langle x|=I
$$

where $\delta\left(x^{\prime}-x\right)$ is the Dirac delta function and $I$ is the identity operator. 
In the same way one can prove that the eigenvalues in $\hat{p}|p\rangle=p|p\rangle$ span a continuum from $-\infty$ to $+\infty$ and that the eigenvectors satisfy:

$$
\left\langle p^{\prime} \mid p\right\rangle=\delta\left(p^{\prime}-p\right) \quad \int d p|p\rangle\langle p|=I
$$

\section{2) Representations}

In configuration space one has:

$$
\Phi(x)=\langle x \mid \Phi\rangle=\langle x|\hat{x}| \Psi\rangle=x\langle x \mid \Psi\rangle=x \Psi(x)
$$

and:

$$
\begin{aligned}
\left\langle x^{\prime}|[\hat{x}, \hat{p}]| x\right\rangle & =i \hbar \delta\left(x^{\prime}-x\right)=\left\langle x^{\prime}|\hat{x} \hat{p}-\hat{p} \hat{x}| x\right\rangle \\
& =\int d x^{\prime \prime}\left\langle x^{\prime}|\hat{x}| x^{\prime \prime}\right\rangle\left\langle x^{\prime \prime}|\hat{p}| x\right\rangle-\int d x^{\prime \prime}\left\langle x^{\prime}|\hat{p}| x^{\prime \prime}\right\rangle\left\langle x^{\prime \prime}|\hat{x}| x\right\rangle \\
& =\int d x^{\prime \prime} x^{\prime \prime} \delta\left(x^{\prime}-x^{\prime \prime}\right)\left\langle x^{\prime \prime}|\hat{p}| x\right\rangle-\int d x^{\prime \prime}\left\langle x^{\prime}|\hat{p}| x^{\prime \prime}\right\rangle x \delta\left(x^{\prime \prime}-x\right) \\
& =x^{\prime}\left\langle x^{\prime}|\hat{p}| x\right\rangle-x\left\langle x^{\prime}|\hat{p}| x\right\rangle=\left(x^{\prime}-x\right)\left\langle x^{\prime}|\hat{p}| x\right\rangle \\
& \left\langle x^{\prime}|\hat{p}| x\right\rangle=\frac{i \hbar \delta\left(x^{\prime}-x\right)}{\left(x^{\prime}-x\right)} \Longrightarrow_{x^{\prime} \rightarrow x} i \hbar \frac{d}{d x^{\prime}} \delta\left(x^{\prime}-x\right)
\end{aligned}
$$

Then:

$$
\begin{aligned}
\Phi(x) & =\langle x \mid \Phi\rangle=\langle x|\hat{p}| \Psi\rangle= \\
& =\int d x^{\prime}\left\langle x|\hat{p}| x^{\prime}\right\rangle\left\langle x^{\prime} \mid \Psi\right\rangle=i \hbar \int d x^{\prime}\left[\frac{d}{d x^{\prime}} \delta\left(x^{\prime}-x\right)\right]\left\langle x^{\prime} \mid \Psi\right\rangle \\
& =\left[\delta\left(x^{\prime}-x\right) \Psi\left(x^{\prime}\right)\right]_{-\infty}^{+\infty}-i \hbar \int d x^{\prime} \delta\left(x^{\prime}-x\right) \frac{d}{d x^{\prime}} \Psi\left(x^{\prime}\right)=-i \hbar \frac{d}{d x} \Psi(x)
\end{aligned}
$$

i.e., the representation in configuration space of the vector $|\Phi\rangle=\hat{p}|\Psi\rangle$ is obtained by taking the derivative of the representation of the vector $|\Psi\rangle$, while the representation in configuration space of the vector $|\Theta\rangle=\hat{x}|\Psi\rangle$ is obtained multiplying by $x$ the representation of $|\Psi\rangle$.

To conclude, in configuration space one has:

$$
\hat{x} \Longrightarrow x \quad \hat{p} \Longrightarrow-i \hbar \frac{d}{d x}
$$

and in the same way in momentum space:

$$
\hat{x} \Longrightarrow i \hbar \frac{d}{d p} \quad \hat{p} \Longrightarrow p
$$

\section{3) Transformation between representations}


Consider:

$$
\langle x|[\hat{x}, \hat{p}]| p\rangle=i \hbar\langle x \mid p\rangle
$$

Developing:

$$
\begin{aligned}
\langle x|[\hat{x}, \hat{p}]| p\rangle= & \langle x|\hat{x} \hat{p}| p\rangle-\langle x|\hat{p} \hat{x}| p\rangle \\
= & x p\langle x \mid p\rangle-\int d x^{\prime}\left\langle x|\hat{p}| x^{\prime}\right\rangle\left\langle x^{\prime}|\hat{x}| p\right\rangle \\
= & x p\langle x \mid p\rangle-i \hbar \int d x^{\prime}\left[\frac{d}{d x^{\prime}} \delta\left(x^{\prime}-x\right)\right] x^{\prime}\left\langle x^{\prime} \mid p\right\rangle \\
= & \left.x p\langle x \mid p\rangle-i \hbar\left[\delta\left(x^{\prime}-x\right)\right] x^{\prime}\left\langle x^{\prime} \mid p\right\rangle\right]_{-\infty}^{+\infty} \\
& +i \hbar \int d x^{\prime} \delta\left(x^{\prime}-x\right) \frac{d}{d x^{\prime}}\left[x^{\prime}\left\langle x^{\prime} \mid p\right\rangle\right] \\
= & x p\langle x \mid p\rangle+i \hbar\left[\langle x \mid p\rangle+i \hbar x \frac{d}{d x}\langle x \mid p\rangle\right]
\end{aligned}
$$

Substituting in Eq.(A.10) one obtains:

$$
\begin{gathered}
x p\langle x \mid p\rangle+i \hbar\left[\langle x \mid p\rangle+i \hbar x \frac{d}{d x}\langle x \mid p\rangle\right]=i \hbar\langle x \mid p\rangle \\
i \hbar \frac{d}{d x}\langle x \mid p\rangle=-p\langle x \mid p\rangle
\end{gathered}
$$

which is satisfied if:

$$
\langle x \mid p\rangle=C e^{i p x / \hbar} \quad\langle p \mid x\rangle=C^{*} e^{-i p x / \hbar}
$$

Finally:

$$
\Phi(p)=\langle p \mid \Psi\rangle=\int d x\langle p \mid x\rangle\langle x \mid \Psi\rangle=C^{*} \int d x e^{-i p x / \hbar} \Psi(x)
$$

and:

$$
\Psi(x)=\langle x \mid \Psi\rangle=\int d p\langle x \mid p\rangle\langle p \mid \Psi\rangle=C \int d x e^{i p x / \hbar} \Phi(p)
$$

i.e., the representations of the state vector in the configuration and momentum spaces are Fourier transforms of each other. To preserve normalization one requires $C=C^{*}=1 / \sqrt{2 \pi \hbar}$.

4) Uncertainty relation

Consider the state vectors

$$
|\Phi\rangle=(\hat{x}-\langle x\rangle)|\Psi\rangle \quad \text { and } \quad|\Xi\rangle=(\hat{p}-\langle p\rangle)|\Psi\rangle
$$

Then

$$
\langle\Phi \mid \Phi\rangle=\left\langle\Psi\left|\hat{x}^{2}\right| \Psi\right\rangle-\langle\Psi|\hat{x}| \Psi\rangle^{2}=(\Delta x)_{\Psi}^{2}
$$


and

$$
\langle\Xi \mid \Xi\rangle=\left\langle\Psi\left|\hat{p}^{2}\right| \Psi\right\rangle-\langle\Psi|\hat{p}| \Psi\rangle^{2}=(\Delta p)_{\Psi}^{2}
$$

By Schawrz inequality one has

$$
\begin{aligned}
\langle\Phi \mid \Phi\rangle\langle\Xi \mid \Xi\rangle & \geq|\langle\Phi \mid \Xi\rangle|^{2}= \\
& =\left|\left\langle\Psi\left|\frac{1}{2}[\hat{x}, \hat{p}]+\frac{1}{2}\{\hat{x}, \hat{p}\}-\langle x\rangle\langle p\rangle\right| \Psi\right\rangle\right|^{2} \geq \\
& \geq\left|\left\langle\Psi\left|\frac{1}{2}[\hat{x}, \hat{p}]\right| \Psi\right\rangle\right|^{2}=(\hbar / 2)^{2}
\end{aligned}
$$

Finally

$$
(\Delta x)_{\Psi}(\Delta p)_{\Psi} \geq \hbar / 2
$$

\section{References}

[1] Pauli, W., "The general principles of quantum mechanics", SpringerVerlag, Berlin Heidelberg, footnote p.63 (1980),

[2] Dirac, P.A.M., "The principles of quantum mechanics" (4th ed.), Oxford, Clarendon Press (1958).

[3] Muga, J.G., R. Sala Mayato and I.L. Egusquiza, "Introduction", in J.G. Muga, R. Sala Mayato, I.L. Egusquiza (eds.) "Time in Quantum Mechanics", pp 1-28 Berlin Springer (2002); reprinted as "Time in Quantum Mechanics, Vol. 1", Lect. Notes Phys. 734, Springer-Verlag, Berlin (2008)

[4] Muga, J.G., A. Ruschhaupt and A. del Campo (eds), "Time in Quantum Mechanics, Vol. 2", Lect. Notes Phys. 789, Springer-Verlag, Berlin (2009).

[5] Bauer, M. and P.A. Mello, "The time-energy uncertainty relation", Ann.Phys. 111, 38-60 (1978)

[6] Busch, P., "The time-energy uncertainty relation", chapter 3 in J.G. Muga, R. Sala Mayato, I.L. Egusquiza (eds.) "Time in Quantum Mechanics", Berlin Springer (2002); revised version arXiv:quant-ph/0105049v3 (2007)

[7] Briggs, J., "A derivation of the time-energy uncertainty relation", Journal of Physics: Conference Series 99, 012002 (2008)

[8] Hilgevoord, J., "Time in Qhantum Mrchanics", Am. J. Phys. 70, 301-306 (2002)

[9] Galapon, E.A., "Post-Pauli's Theorem Emerging Perspective on Time In Quantum Mechanics", Chapter 3 in Lect. Notes Phys. 789, 25-63 (2009)

[10] Boykin, T.B., N. Kharche and G. Klimeck, "Evolution time and energy uncertainty", Eur. J. Phys. 28, 673-678 (2007) 
[11] Bauer, M., "A dynamical time operator in Dirac's relativistic quantum mechanics", Int.J.Mod.Phys. A 29, 1450036 (2014)

[12] Briggs, J.S. and Jan M. Rost, "Time dependence in quantum mechanics", Eur.Phys.J.10, (2000); "On the Derivation of the Time-dependent Equation of Schrödinger", Foundations of Physics 31, (2001)

[13] Moreva, E. et al., "Time from quantum entanglement: an experimental illustration", arXiv:1310.4691v1 [quant-ph] (2013); "The time as an emergent property of quantum mechanics, a synthetic description of a first experimental approach", J.Phys.: Conference Series 626, 012019 (2015)

[14] Thaller, B., "The Dirac Equation", Springer-Velag, Berlin Heidelberg New York (1992)

[15] Greiner, W., "Relativistic Quantum Mechanics - Wave equations", (3 ${ }^{\mathrm{d}}$ ed.) Springer, Berlin Heidelberg New York (2000)

[16] Messiah, A., "Quantum Mechanics", Vol.I, p. 442, North-Holland Publishing Company, Amsterdam, and John Wiley\&Sons, New York London Sidney, 4th printing (1966)

[17] Jordan, T.J., "Linear operators for quantum mechanics", John Wiley \& Sons, Inc. (1969)

[18] de Broglie, L., Ph.D. thesis; Ann. Phys.,Ser. 10 ${ }^{\mathrm{e}}$, t. III (1925). English translation reprinted in Ann.Fond.Louis de Broglie 17, p. 92 (1992)

[19] Baylis, W.E., "De Broglie waves as an effect of clock desynchronization", Can.J.Phys. 85, pp.1317-1323 (2007)

[20] Ferber, R., "A Missing Link: What is behind de Broglie's "periodic phenomenon"?, Found.Phys.Lett. 9, 575-586 (1996)

[21] Lan, S.Y. et al., "A Clock Directly Linking Time to a Particle Mass", Science 339, 554-557 (2013)

[22] Catillon, P. et al., "A Search for the de Broglie Particle Internal Clock by means of Electron Channeling", Found.Phys. 38, 659-664 (2008)

[23] Cserti, J. and G. David, "Unified description of Zitterbewegung for spintronic, graphene, and superconducting systems", Phys.Rev. B 74, 172305 (2006)

[24] Gerritsma, R. et al., "Quantum simulation of the Dirac equation", Nature 463, pp.68-71 (2010)

[25] LeBlanc, L.J. et al., "Direct observation of Zitterbewegung in a BoseEinstein condensate", New J.Phys. 15, 073011 (2013) 
[26] Anderson, E., "The problem of time in quantum gravity", Ann.Phys. (Berlin) 524, 757-786 (2012) and references therein; also arXiv:1009.2157v3 [gr-qc]

[27] Isham, C.J., "Canonical Quantum Gravity and the Problem of Time", arXiv:gr-qc/9210011v1, (1992); "Prima Facie Questions in Quantum Gravity", arXiv:gr-qc/9310031v1 (1993)

[28] Butterfield, J. and C.J. Isham, "On the Emergence of Time in Quantum Gravity", arXiv:gr-qc/9901024v1 (1999)

[29] Bauer, M., "Quantum Gravity and a Time Operator in Relativistic Quantum Mechanics", arXiv:gr-qc/1605.01659 (2016) 